\title{
A New Model of Counterion Condensation in Polyelectrolyte Solutions II. Application to Potentiometric Titration of Weak Polyacids
}

\author{
Mitsuru SATOH* and Jiro KomiYAmA \\ Department of Polymer Science, Tokyo Institute of Technology, \\ Ookayama, Meguro-ku, Tokyo 152, Japan
}

(Received April 2, 1987)

\begin{abstract}
The apparent dissociation constants, $\mathrm{p} K_{\mathrm{a}}$, of weak polyacids in aqueous salt solutions are estimated as a function of the degree of dissociation, $\alpha$, by our new model for counterion condensation (Intermediate Model, IMM) and our corrected previous model (CPM). Both models predict smooth titration curves free from a break point which is a defect in the prediction by Manning theory. Titration curves obtained by CPM show a maximum, indicating that CPM overestimates the degree of condensation with increasing $\alpha$. Sigmoidal titration curves are predicted by IMM; the underestimation for a lower $\alpha$ region of poly(acrylic acid) is corrected through effective charge densities estimated from the counterion activity data, and that observed for the higher $\alpha$ region is interpreted as caused by hydration changes of the polyion with $\alpha$.

KEY WORDS Potentiometric Titration / Polyelectrolyte / Counterion Condensation /
\end{abstract}

In our preceding study (Part $\left.I^{1}\right)$, we have examined our modified models for counterion condensation (Corrected Previous Model (CPM) and Intermediate Model (IMM)) by comparing with previous condensation theories by Manning ${ }^{2}$ and Iwasa et al. $^{3}$ and with experimental data for the counterion activity coefficients, $\gamma_{c}{ }^{\prime}$. In the present study, we apply IMM and CPM to potentiometric titration of weak polyacids. The apparent dissociation constant, $K_{\mathrm{a}}$, is estimated as a function of the charge density or the degree of dissociation, $\alpha$, by taking account of the counterion condensation. Manning has already derived equations for $\mathrm{p} K_{\mathrm{a}}\left(=-\log K_{\mathrm{a}}\right)$ based on his condensation theory. ${ }^{5}$ The predicted titration curve, $\mathrm{p} K_{\mathrm{a}}$ vs. $\alpha$, however, shows a break at the critical charge density above which the counterion condensation will take place. This discontinuity is caused by an artifact that the volume term, $V_{\mathrm{p}}$, for the condensed phase abruptly appears at the critical point in the derivation of the equation. As described in part $\mathrm{I}$, we have adopted models devoid of $V_{\mathrm{p}}$, IMM and CPM, since it is the use of $V_{\mathrm{p}}$ term, i.e., the assumption on the presence of the condensed phase that prevents Manning theory from estimating the ionic strength dependence of the condensation. As shown below, our models (IMM and CPM) do not give such a discontinuity in the theoretical titration curves. This is also because both models assume counterion binding modes which do not involve such a volume term; in CPM the mixing entropy of the condensed counterions is neglected and in IMM the binding position of the condensed counterions is assigned to charged groups.

In the following sections, theoretical equations for the titration curve are derived based on IMM and CPM and the results are compared with experimental data. Effects of polymer conformation and hydration changes

* To whom correspondence should be addressed. 
with increasing $\alpha$ are also discussed.

\section{EQUATIONS FOR POTENTIOMETRIC TITRATION}

We treat $\mathrm{pH}$ titration of a weak polyacid in aqueous salt solution. Polymer model and assumptions are the same as in Part $\mathrm{I}^{1}{ }^{1}$ The free energy $\Delta G$ of the system in which protonation and counterion condensation to the polymer charges occur is expressed as

$$
\begin{aligned}
\Delta G / R T= & \left\{C_{\mathrm{H}}-(1-\alpha) C_{\mathrm{p}}\right\} \\
& \times\left[\ln \left\{C_{\mathrm{H}}-(1-\alpha) C_{\mathrm{p}}\right\}+\ln \gamma_{\mathrm{H}}^{\mathrm{ii}}\right] \\
& +\left\{C_{\mathrm{s}}+(1-\theta) \alpha C_{\mathrm{p}}\right\} \\
& \times\left[\ln \left\{C_{\mathrm{s}}+(1-\theta) \alpha C_{\mathrm{p}}\right\}+\ln \gamma_{\mathrm{c}}^{\mathrm{ii}}\right] \\
& +C_{\mathrm{w}} \ln a_{\mathrm{w}}+F_{\mathrm{ex}} / R T \\
& +C_{\mathrm{p}}\{\alpha \ln \alpha+(1-\alpha) \ln (1-\alpha)\} \\
& +\alpha C_{\mathrm{p}}\{\theta \ln \theta+(1-\theta) \ln (1-\theta)\} \\
& +(1-\alpha) C_{\mathrm{p}} \ln K_{\mathrm{a}}^{\circ}
\end{aligned}
$$

where $C$ is molar concentration, $\gamma^{\mathrm{ii}}$ 's are activity coefficients for small ion-small ion interactions, $a_{\mathrm{w}}$ is water activity given by the mole fraction, and $K_{\mathrm{a}}^{\circ}$ is the dissociation constant of the monomer acid. Subscripts, $\mathbf{H}$, $\mathrm{p}, \mathrm{s}, \mathrm{c}$ and $\mathrm{w}$ mean proton, polymer, added salt, counterion and water, respectively. $F_{\text {ex }}$, the excess free energy for electrostatic interactions between polymer charges and between small ion and polyion, is estimated with the screened coulombic potential of Debye-Hückel;

$$
\begin{aligned}
& F_{\mathrm{ex}} / R T=\alpha^{2} C_{\mathrm{p}} \xi(1-\theta)^{2} \sum_{m=1}^{N-1}(1-m / N) \\
& \times \exp (-\kappa m b / \alpha) / m \\
& \kappa^{2}=8 \pi \times 10^{3} N_{\mathrm{Av}}\left(e^{2} / D k T\right) I \\
& I=1 / 2 \times\left(2 C_{\mathrm{s}}+C_{\mathrm{H}}-(1-\alpha) C_{\mathrm{p}}+(1-\theta) \alpha C_{\mathrm{p}}\right)
\end{aligned}
$$

where $\xi$ is the charge density parameter given as $e^{2} / D k T b, e$ is the electronic charge, $D$ is the dielectric constant of the bulk solvent, $T$ is the Kelvin temperature, and $b$ is the axial spacing of the functional groups. $N$ is the number of charges on a polyion, $N_{\mathrm{Av}}$ is the Avogadro number and $I$ is the ionic strength. In eq 1 , terms which do not contain $\theta$ and $\alpha$ are omitted. Since the degree of condensation per polymer functional group is given by $\alpha \theta$, the condition for the free energy minimization is as follows

$$
\{\partial(\Delta G / R T) / \partial \alpha\}_{\alpha \theta}=0
$$

and

$$
\{\partial(\Delta G / R T) / \partial \alpha \theta\}_{\alpha}=0
$$

Equation $3 b$ is the condition to determine the degree of counterion condensation. Therefore a theoretical expression for $\mathrm{p} K_{\mathrm{a}}$ is obtained by eq $3 a$ in which $\theta$ values satisfying eq $3 b$ are substituted;

$$
\begin{aligned}
\mathrm{p} K_{\mathrm{a}}= & \mathrm{pH}+\log (\alpha / 1-\alpha) \\
= & \mathrm{p} K_{\mathrm{a}}^{\circ}+0.434\left(\partial\left(F_{\mathrm{ex}} / C_{\mathrm{p}} R T\right) / \partial \alpha\right. \\
& \left.+\ln (1-\theta)-\ln \gamma_{\mathrm{H}}\right)
\end{aligned}
$$

In the equation, $\ln (1-\theta)$ term expresses the contribution of the mixing entropy of the condensed counterions to the free energy change of the system due to increment of the charge density. $\gamma_{\mathrm{H}}$, the activity coefficient of proton for electrostatic interaction with polymer charges, appears because the partial differentiation of $F_{\mathrm{ex}} / C_{\mathrm{p}} R T$ produces $\ln \gamma_{\mathrm{H}}$ term. The partial derivative is numerically obtained because $F_{\text {ex }}$ term contains a summation on $N$ which is a function of $\alpha . \theta$ values in eq 4 can be estimated separately by the method described in our preceding paper (Part $\left.\mathrm{I}^{1}\right)$.

The equation based on CPM is as follows

$$
\mathrm{p} K_{\mathrm{a}}=\mathrm{p} K_{\mathrm{a}}^{\circ}+0.434\left(\partial\left(F_{\mathrm{ex}} / C_{\mathrm{p}} R T\right) / \partial \alpha-\ln \gamma_{\mathrm{H}}\right)
$$




\section{COMPARISON OF $\mathrm{p} K_{\mathrm{a}}$ ESTIMATED BY IMM AND CPM}

In Table I, theoretical values of $\mathrm{p} K_{\mathrm{a}}$ calculated by eq 4 and 5 are listed with those by Manning theory ${ }^{4}$ and experimental values obtained by Olander and Holtzer for poly(DLglutamic acid) (PDLG). ${ }^{5}$ A marked difference can be seen between predictions by our models and Manning theory; the latter predicts a discontinuous change in $\mathrm{p} K_{\mathrm{a}}$ values at $\alpha=0.5$. According to Manning, the discontinuity is caused by the divergence of $\ln V_{\mathrm{p}}\left(V_{\mathrm{p}}\right.$, volume of the condensed phase) at the critical charge density. In other words, the discontinuity is inherent in the two phase model. On the contrary, IMM and CPM predict continuous change of $\mathrm{p} K_{\mathrm{a}}$ at the onset of counterion condensation. Though under the experimental conditions used in Table I, IMM predicts nonzero values for $\theta$ even at the lowest charge density $(\alpha=0.1), \mathrm{p} K_{\mathrm{a}}$ for $\theta=0$ can be calculated just by substituting $\theta=0$ to eq 4 .

CPM predicts another unrealistic behavior of $\mathrm{p} K_{\mathrm{a}}$ as seen in Table I. $\mathrm{p} K_{\mathrm{a}}$ decreases with $\alpha$ above $\alpha=0.7$. Such decrease in $\mathrm{p} K_{\mathrm{a}}$ with increasing $\alpha$ has not been observed except for hydrophobic polyacids ${ }^{6,7}$ or biopolymers ${ }^{8,9}$ which are subject to conformational transitions upon neutralization. The cause for the anomaly predicted by CPM (eq 5) may be found in Table II, which lists effective fractional charge, $\alpha^{\prime}(=\alpha(1-\theta))$, of model polyions estimated by IMM, CPM, and Manning theory. Though $\alpha^{\prime}$ estimated by IMM slightly decreases with $\alpha$ in higher $\alpha$ region, the dependence of $\alpha^{\prime}$ on $\alpha$ is comparable to that by Manning theory which predicts a constant $\alpha^{\prime}$ above the critical $\alpha$ values. On the other hand, $\alpha^{\prime}$ estimated by CPM significantly decreases in $\alpha$ region where $\theta>0$, indicating that $\theta$ values estimated with CPM increase with the charge

Table I. Experimental and theoretical $\mathrm{p} K_{\mathrm{a}}$ values $^{\mathrm{a}}$ for PDLG

\begin{tabular}{|c|c|c|c|c|c|c|c|c|}
\hline \multirow{2}{*}{$\alpha$} & \multicolumn{4}{|c|}{$C_{\mathrm{s}}=0.01 \mathrm{M}$} & \multicolumn{4}{|c|}{$C_{\mathrm{s}}=0.05 \mathrm{M}$} \\
\hline & Exptl & IMM & $\mathrm{CPM}$ & Manning & Exptl & IMM & CPM & Manning \\
\hline 1.0 & 6.37 & $\begin{array}{c}6.36 \\
(0.477)\end{array}$ & $\begin{array}{l}6.63 \\
(0.478)\end{array}$ & 6.65 & 5.78 & $\begin{array}{c}5.74 \\
(0.471)\end{array}$ & $\begin{array}{c}5.98 \\
(0.483)\end{array}$ & 5.83 \\
\hline 0.9 & 6.26 & $\begin{array}{c}6.31 \\
(0.422)\end{array}$ & $\begin{array}{c}6.67 \\
(0.391)\end{array}$ & 6.54 & 5.66 & $\begin{array}{c}5.70 \\
(0.417)\end{array}$ & $\begin{array}{c}6.01 \\
(0.388)\end{array}$ & 5.72 \\
\hline 0.8 & 6.10 & $\begin{array}{c}6.26 \\
(0.359)\end{array}$ & $\begin{array}{c}6.72 \\
(0.273)\end{array}$ & 6.40 & 5.56 & $\begin{array}{c}5.64 \\
(0.356)\end{array}$ & $\begin{array}{l}6.07 \\
(0.257)\end{array}$ & 5.58 \\
\hline 0.7 & 6.00 & $\begin{array}{c}6.17 \\
(0.286)\end{array}$ & $\begin{array}{l}6.80 \\
(0.107)\end{array}$ & 6.25 & 5.47 & $\begin{array}{c}5.55 \\
(0.290)\end{array}$ & $\begin{array}{l}6.17 \\
(0.069)\end{array}$ & 5.43 \\
\hline 0.6 & 5.90 & $\begin{array}{c}6.04 \\
(0.208)\end{array}$ & $\begin{array}{c}6.61 \\
(0.0)\end{array}$ & 6.06 & 5.36 & $\begin{array}{c}5.42 \\
(0.221)\end{array}$ & $\begin{array}{c}5.91 \\
(0.0)\end{array}$ & 5.24 \\
\hline 0.5 & 5.76 & $\begin{array}{c}5.84 \\
(0.134)\end{array}$ & $\begin{array}{c}6.14 \\
(0.0)\end{array}$ & $5.83,6.32$ & 5.27 & $\begin{array}{c}5.25 \\
(0.157)\end{array}$ & $\begin{array}{c}5.53 \\
(0.0)\end{array}$ & $5.01,5.56$ \\
\hline 0.4 & 5.60 & $\begin{array}{l}5.57 \\
(0.075)\end{array}$ & $\begin{array}{c}5.70 \\
(0.0)\end{array}$ & 5.83 & 5.15 & $\begin{array}{c}5.04 \\
(0.106)\end{array}$ & $\begin{array}{c}5.19 \\
(0.0)\end{array}$ & 5.21 \\
\hline 0.3 & 5.43 & $\begin{array}{c}5.25 \\
(0.039)\end{array}$ & $\begin{array}{r}5.30 \\
(0.0)\end{array}$ & 5.38 & 5.02 & $\begin{array}{c}4.81 \\
(0.071)\end{array}$ & $\begin{array}{c}4.89 \\
(0.0)\end{array}$ & 4.90 \\
\hline 0.2 & 5.27 & $\begin{array}{c}4.93 \\
(0.020)\end{array}$ & $\begin{array}{c}4.95 \\
(0.0)\end{array}$ & 4.99 & 4.90 & $\begin{array}{l}4.61 \\
(0.050)\end{array}$ & $\begin{array}{c}4.64 \\
(0.0)\end{array}$ & 4.65 \\
\hline 0.1 & 5.02 & $\begin{array}{c}4.67 \\
(0.012)\end{array}$ & $\begin{array}{c}4.68 \\
(0.0)\end{array}$ & 4.69 & 4.74 & $\begin{array}{c}4.47 \\
(0.041)\end{array}$ & $\begin{array}{r}4.49 \\
(0.0)\end{array}$ & 4.49 \\
\hline
\end{tabular}

a Experimental and theoretical values by Manning were obtained from ref 4 . The parenthesized values are $\theta$ values estimated by IMM and CPM. $N, b$, and $C_{\mathrm{p}}$ values used in the calculation are obtained by $N=185 \times \alpha, b=2.5 / \alpha$ $\left(10^{-8} \mathrm{~cm}\right)$, and $C_{\mathrm{p}}=0.02 \times \alpha\left(\right.$ eq $\left.1^{-1}\right)$, respectively. 
Table II. Effective charge fraction $\alpha^{\text {a }}$

\begin{tabular}{|c|c|c|c|c|}
\hline$b\left(10^{-8} \mathrm{~cm}\right)$ & $\alpha$ & IMM & CPM & Manning \\
\hline \multirow[t]{10}{*}{1.0} & 0.1 & 0.095 & 0.100 & 0.100 \\
\hline & 0.2 & 0.142 & 0.173 & 0.140 \\
\hline & 0.3 & 0.147 & 0.143 & 0.140 \\
\hline & 0.4 & 0.146 & 0.127 & 0.140 \\
\hline & 0.5 & 0.143 & 0.117 & 0.140 \\
\hline & 0.6 & 0.141 & 0.110 & 0.140 \\
\hline & 0.7 & 0.139 & 0.105 & 0.140 \\
\hline & 0.8 & 0.137 & 0.100 & 0.140 \\
\hline & 0.9 & 0.136 & 0.097 & 0.140 \\
\hline & 1.0 & 0.135 & 0.094 & 0.140 \\
\hline \multirow[t]{10}{*}{2.0} & 0.1 & 0.098 & 0.100 & 0.100 \\
\hline & 0.2 & 0.190 & 0.200 & 0.200 \\
\hline & 0.3 & 0.254 & 0.300 & 0.280 \\
\hline & 0.4 & 0.282 & 0.341 & 0.280 \\
\hline & 0.5 & 0.291 & 0.306 & 0.280 \\
\hline & 0.6 & 0.292 & 0.282 & 0.280 \\
\hline & 0.7 & 0.291 & 0.265 & 0.280 \\
\hline & 0.8 & 0.289 & 0.251 & 0.280 \\
\hline & 0.9 & 0.287 & 0.240 & 0.280 \\
\hline & 1.0 & 0.285 & 0.231 & 0.280 \\
\hline \multirow[t]{10}{*}{3.0} & 0.1 & 0.099 & 0.100 & 0.100 \\
\hline & 0.2 & 0.195 & 0.200 & 0.200 \\
\hline & 0.3 & 0.284 & 0.300 & 0.300 \\
\hline & 0.4 & 0.354 & 0.400 & 0.400 \\
\hline & 0.5 & 0.398 & 0.500 & 0.420 \\
\hline & 0.6 & 0.420 & 0.509 & 0.420 \\
\hline & 0.7 & 0.431 & 0.472 & 0.420 \\
\hline & 0.8 & 0.435 & 0.443 & 0.420 \\
\hline & 0.9 & 0.436 & 0.420 & 0.420 \\
\hline & 1.0 & 0.436 & 0.402 & 0.420 \\
\hline
\end{tabular}

a $N=1000, C_{\mathrm{s}}=0.01 \mathrm{M}, C_{\mathrm{p}}=0.01 \mathrm{M}$.

density at higher rate than those by IMM and Manning theory. This depression of $\alpha^{\prime}$ leads to the anomalous decrease in $\mathrm{p} K_{\mathrm{a}}$. Thus CPM is inadequate to treat effects of charge density on the condensation. Therefore we will confine ourselves to discussions on IMM in the following sections.

\section{COMPARISON WITH EXPERIMENTAL DATA}

Theoretical $\mathrm{p} K_{\mathrm{a}}$ values estimated by IMM (eq 4) agree well with the experimental values for PDLG as shown in Table I. The largest deviations are $0.35 \mathrm{p} K$ unit for $C_{\mathrm{s}}=0.01 \mathrm{M}$ and

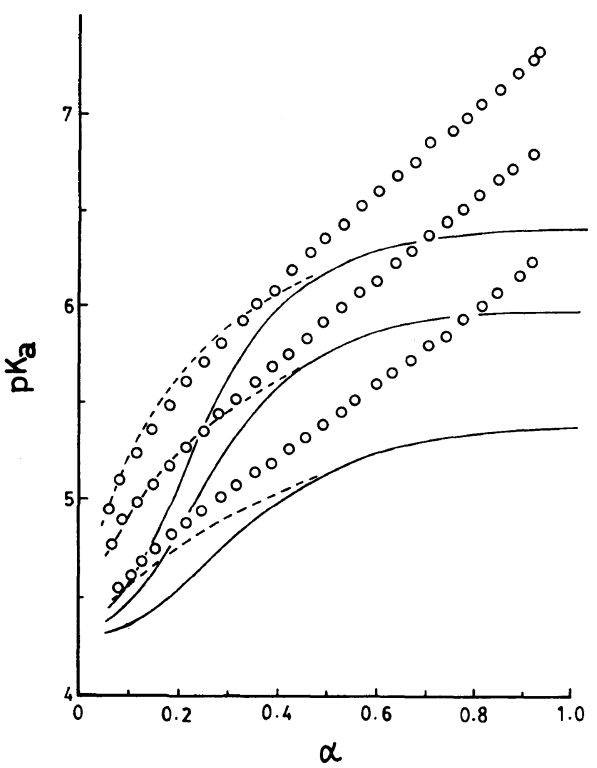

Figure 1. Comparison of experimental $\mathrm{p} K_{\mathrm{a}}$ values for PAA of Nagasawa et al. $^{7}$ with theoretical curves by IMM. $N=2083, b=2.5 \times 10^{-8} \mathrm{~cm}, C_{\mathrm{p}}=0.00829 \mathrm{eq}^{-1}$. Solid curves, IMM and dashed curves, IMM with the correction of the effective charge density. $C_{\mathrm{s}}$ for the experimental data and the theoretical curves are $0.1 \mathrm{M}$, $0.02 \mathrm{M}$, and $0.005 \mathrm{M}$ from bottom to top.

$0.29 \mathrm{pK}$ unit for $C_{\mathrm{s}}=0.05 \mathrm{M}$. This is comparable to the estimation of Manning theory. However, the dependence of $\mathrm{p} K_{\mathrm{a}}$ on $\alpha$ estimated by IMM is rather different from the experimental result; the theoretical values increase with $\alpha$ in a sigmoidal shape. This is because of two reasons; at the lowest $\alpha$ region, IMM based upon the linear charge array model tends to predict only slight increase in the electrostatic interaction between polymer charges with increasing $\alpha$. At the higher $\alpha$ region, IMM predicts the saturation of the interaction due to the counterion condensation. A similar trend is observed in the prediction by Manning theory, although the latter estimates steeper increase of $\mathrm{p} K_{\mathrm{a}}$ than the former both for $\alpha \leqq 0.5$ and $\alpha \geqq 0.5$. The sigmoidal dependence of $\mathrm{p} K_{\mathrm{a}}$ on $\alpha$ is more pronounced for poly(acrylic acid) (PAA) which has higher charge density than PDLG, as shown in Figure 1. The experimental data 


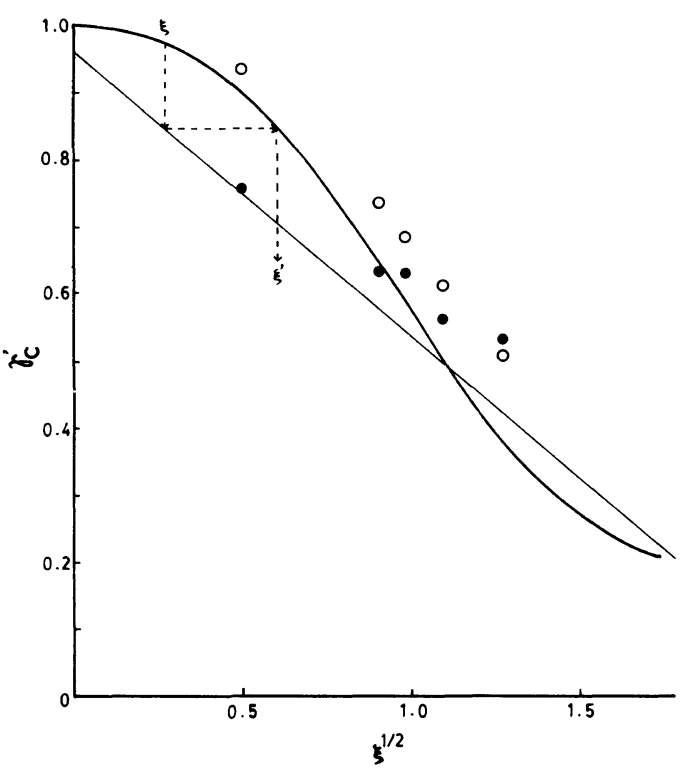

Figure 2. Dependence of experimental and theoretical $\gamma_{c}{ }^{\prime}$ on $\xi^{1 / 2}$. Thin line, empirical relation of $\gamma_{c}{ }^{\prime}$ and $\xi^{1 / 2}$ obtained by Kowblansky and Zema ${ }^{11}$ for poly(acrylic acid/acrylamide) copolymers and thick curve, IMM, $N=\infty, C_{\mathrm{p}}=0.0045 \mathrm{eq} \mathrm{l}^{-1}$. (O) experimental data obtained by Joshi and $\mathrm{Kwak}^{12}$ for sodium pectinates, $(\mathrm{O})$ IMM, $C_{\mathrm{p}}=0.01 \mathrm{eq} \mathrm{l}^{-1}, C_{\mathrm{s}}=0.00172 \mathrm{M} ; N=162,160$, 161,160 and $17 ; b=4.38,5.95,7.36,8.71$, and $28.56 \times 10^{-8} \mathrm{~cm}$, respectively. The arrows show how to obtain effective charge density $\xi^{\prime}$ for a polyacrylic acid with a charge density $\xi$.

plotted in the figure are those obtained by Nagasawa et al. ${ }^{6}$ Similar deviations in the theoretical and experimental values are noted on counterion activity coefficients of polyions with low charge densities. ${ }^{10,11}$ Kowblansky and $\mathrm{Zema}^{10}$ determined sodium ion activity coefficients in aqueous solutions of fully neutralized poly(acrylic acid/acrylamide) copolymers having a wide range of charge densities. The authors found that the activity coefficients can be expressed as a linear function of $\xi^{1 / 2}$. The empirical expression is given by the following equation

$$
\gamma_{\mathrm{c}}{ }^{\prime}=0.96-0.42 \xi^{1 / 2}
$$

in the absence of added salts. The linear relationship is plotted in Figure 2 to compare with the theoretical curve by IMM. Judged from the viscosity data, the copolymers have quite high molecular weights. Therefore, theoretical $\gamma_{c}{ }^{\prime}$ values was calculated by using eq 13 in our preceding paper (part I) which expresses $F_{\text {ex }} / R T$ for $N=\infty$. As seen from Figure 2, the theoretical values of $\gamma_{c}{ }^{\prime}$ are significantly overestimated at lower charge densities $(\xi<1)$, whereas the agreements are relatively good at higher charge densities $(\xi>1)$. Figure 2 contains another example for comparison of theoretical $\gamma_{c}{ }^{\prime}$ values with experimental ones; filled circles represent $\gamma_{c}{ }^{\prime}$ values of sodium pectinates with different charge densities in the presence of $\mathrm{NaCl}$, reported by Joshi and Kwak. ${ }^{11}$ In this case too, the difference of the theoretical values with the experimental data becomes larger with decreasing $\xi^{1 / 2}$. Joshi and Kwak have also found that only for sodium pectinates with low charge densities $(\xi=0.82$ and 0.25$)$ $\gamma_{c}{ }^{\prime}$ values estimated by condensation theories are appreciably larger than the experimental data. The authors attributed the large discrepancy found for the polymer of $\xi=0.25$, to the increment of effective charge density caused from some coiled conformation of the polymer chain at such a low charge density. Their interpretation seems to be applied for the present case; in the lower $\alpha$ region $(\leqq 0.4)$, the coiled conformation and a resultant increased effective charge density of the polymer will lead to underestimations of $\mathrm{p} K_{\mathrm{a}}$ values obtained by IMM, since the higher charge density, the more difficult the dissociation. Assuming this is the case, the theoretical curves for $\alpha \leqq 0.4$ in Figure 1 are corrected by using effective charge densities, values at which the same $\gamma_{c}{ }^{\prime}$ values as experimental ones are theoretically predicted (see arrows in Figure 2). For example, corrected b values (and the original values) at $\alpha=0.1,0.2$, 0.3 , and 0.4 are $11.7(25.0), 8.53$ (12.5), 7.07 (8.33) and $6.12(6.25) \times 10^{-8} \mathrm{~cm}$, respectively. These values and the original values estimated by $2.5 \times 10^{-8} \mathrm{~cm} / \alpha$ used for $\alpha \geqq 0.5$ are to be substituted in eq (2a). The theoretical curves 
thus obtained (dashed curves in Figure 1) can explain the dependence of $\mathrm{p} K_{\mathrm{a}}$ on $\alpha$ much better than before. The correction appears less effective for $C_{\mathrm{s}}=0.1 \mathrm{M}$. This may be because the higher the ionic strength, the more the deviation of polymer charge density from $\xi$ becomes thruugh the polymer chain coiling. Thus eq 4 based upon IMM can predict the dependence of $\mathrm{p} K_{\mathrm{a}}$ on $\alpha$ for polyions with low charge densities if they are properly corrected to the effective values. However, it should be noted that this apparent success in correcting the effective charge density indicates a limited applicability of the linear charge array model adopted by the condensation theories.

The corrected theoretical curves still underestimate the experimental $\mathrm{p} K_{\mathrm{a}}$ at higher $\alpha$ region $(\alpha \geqq 0.5)$. This deviation does not seem to be caused by long-range electrostatic factors. Figure 3 shows that the electrostatic interactions are properly evaluated by IMM. In the figure, the dependences of $\mathrm{p} K_{\mathrm{a}}$ of PAA on $C_{\mathrm{p}}{ }^{1 / 2}$, which have been empirically found linear by Nagasawa et al., ${ }^{6}$ are compared with those by eq 4 . The $\mathrm{p} K_{\mathrm{a}}$ values measured at $C_{\mathrm{s}}=0.1 \mathrm{M}$ decrease only slightly with $C_{\mathrm{p}}{ }^{1 / 2}$, while at $C_{\mathrm{s}}=0.005 \mathrm{M}$ the dependence is much enhanced except for $\alpha=0.1$. It is easily seen in the figure that the theoretical lines qualitatively reproduce such experimentally observed trends for the ionic strength dependence of $\mathrm{p} K_{\mathrm{a}}$. Therefore we attribute the discrepancy between the experimental and the theoretical $\mathrm{p} K_{\mathrm{a}}$ values at higher $\alpha$, to the other factors in the free energy change for the dissociation process of PAA. The factors may consist of conformational changes, hydrogen bonding between carboxyl groups and hydration changes with increasing $\alpha$. The effect of the first one seems to be minor because polymer conformations at high charge densities can be approximated by the linear charge array model, i.e., theoretical $\gamma_{\mathrm{c}}{ }^{\prime}$ values by IMM for PAANa of $\xi>1$ are in good agreement with experimental values as shown in Figure 2. It is well known that the second factor plays an

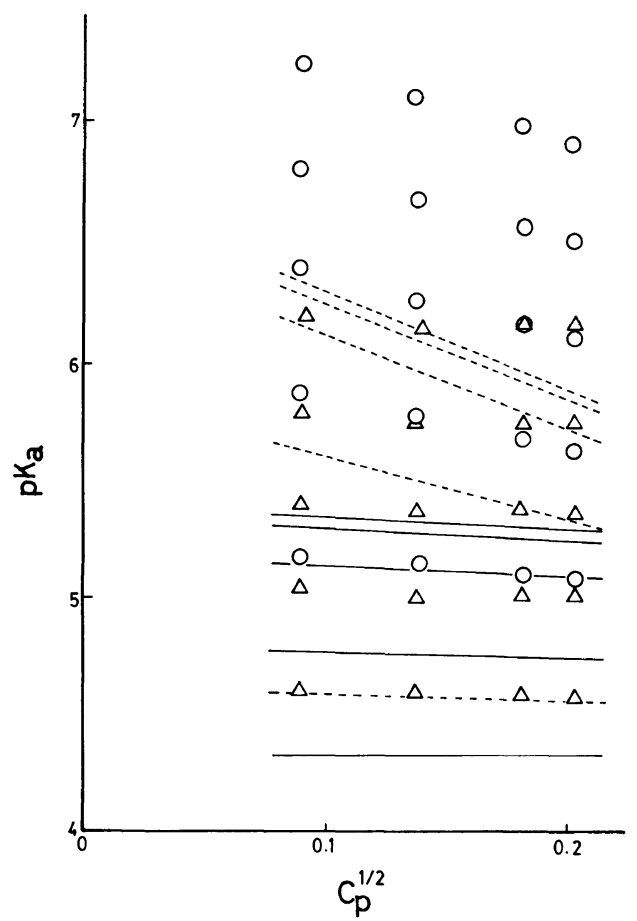

Figure 3. Comparison of the dependence of the experimental $\mathrm{p} K_{\mathrm{a}}$ for PAA of Nagasawa et al. ${ }^{7}$ on $C_{\mathrm{p}}{ }^{1 / 2}$ with the theoretical prediction by IMM. (O) $C_{\mathrm{s}}=$ $0.005 \mathrm{M},(\triangle) 0.1 \mathrm{M}$. Dashed lines, IMM for $C_{\mathrm{s}}=$ $0.005 \mathrm{M}$ and solid lines, IMM for $C_{\mathrm{s}}=0.1 \mathrm{M} . \alpha=0.1$, $0.3,0.5,0.7$, and 0.9 from bottom to top.

important role in determining $\mathrm{p} K$ values of some dibasic acids and diacidic bases. Polyacids and polybases such as maleic acid copolymers and polyvinylamine also exhibit extraordinarily large changes in $\mathrm{p} K_{\mathrm{a}}$ (ca. by $5 \mathrm{pK}$ units). ${ }^{12,13}$ This has been attributed partially to the hydrogen bonding between the functional groups. However the contribution of the hydrogen bonding to the $\mathrm{p} K_{\mathrm{a}}$ values of PAA is not apparent in the smooth titration curves, the overall changes of which are $3 \mathrm{pK}$ units at most. On the other hand, the last factor may exert a substantial effect on the $\mathrm{p} K_{\mathrm{a}}$ values; Ikegami ${ }^{14}$ made refractivity measurements on sodium polyacrylate solutions and calculated the molar volume change by protonation. Figure 6 of his study shows that the molar volume change slightly $(c a .10 \%)$ de- 
creases with increasing $\alpha$. We have also found that degrees of hydration of sodium and cesium polyacrylates, which were estimated by molar compressibility data, decrease with increasing $\alpha(\mathrm{ca} .17 \%$ for PAANa and $11 \%$ for PAACs). ${ }^{15}$ These two experimental facts suggest that disturbance in the primary hydration shells of the polyion and/or the counterions proceeds with the counterion condensation. If water molecules hydrating a charged group are removed by the condensed counterions, the equilibrium constant for the dissociation would be lowered than the corresponding value of the monomer acid, $K_{\mathrm{a}}^{\circ}$, because the latter value is contributed by the stabilization of the dissociation species in full or intact hydration. A rough estimation of the free energy for the dehydration may be obtained from gas phase hydration data of alkali halides ${ }^{16}$ enthalpies of dehydration by one water molecule are about 14 and $9 \mathrm{kcal} \mathrm{mol}^{-1}$ for $\mathrm{Na}^{+}$and $\mathrm{I}^{-}$ions, respectively, when the ions are hydrated with 4 water molecules. The value for carboxylate anion seems not to be far from that of $\mathrm{Na}^{+}$since the reorientation time of the hydrated water molecules around the anion and the hydration number are comparable to those for $\mathrm{Na}^{+} \cdot{ }^{17-19}$ In Table III, differences of the experimental and the theoretical $\mathrm{p} K_{\mathrm{a}}$ values $\left(\Delta \mathrm{p} K_{\mathrm{a}}\right)$ and those of the corresponding free energies $\left(\Delta G^{\prime}\right)$ are shown for PAA and PDLG. The $\Delta G^{\prime}$ values are much less than the values estimated in the above, i.e., $\sim 10 \mathrm{kcal} \mathrm{mol}^{-1}$. This suggests that slight deviation from an optimum orientation of the hydrated water molecules is caused by the counterion condensation. Such perturbation in the hydration is likely to occur for water molecules separating the condensed counterions and the polymer charges. The value for PAA at $\alpha=0.9,1.1 \mathrm{kcal} \mathrm{mol}^{-1}$, can be compared to that estimated for polyvinylamine (PVA) hydroperchlorate by Lewis et al. ${ }^{13}$ The authors have determined the thermodynamic parameters, $\Delta G, \Delta \mathrm{H}$ and $\Delta S$ for dissociation of PVA by calorimetric and potentiometric tit-
Table III. $\Delta \mathrm{p} K_{\mathrm{a}}$ and corresponding $\Delta G^{\prime}$ for PAA and PDLG

\begin{tabular}{|c|c|c|c|c|}
\hline \multirow{2}{*}{ Polymer } & \multirow{2}{*}{$\alpha$} & \multirow{2}{*}{$\frac{C_{\mathrm{s}}}{\mathrm{M}}$} & \multirow{2}{*}{$\Delta \mathrm{p} K_{\mathrm{a}}^{\mathrm{a}}$} & \multirow{2}{*}{$\frac{\Delta G^{\prime}}{\mathrm{kcal} \mathrm{mol}^{-1}}$} \\
\hline & & & & \\
\hline \multirow[t]{6}{*}{ PAA } & 0.9 & 0.1 & 0.78 & 1.1 \\
\hline & & 0.02 & 0.78 & 1.1 \\
\hline & & 0.005 & 0.81 & 1.1 \\
\hline & 0.6 & 0.1 & 0.35 & 0.48 \\
\hline & & 0.02 & 0.26 & 0.36 \\
\hline & & 0.005 & 0.28 & 0.39 \\
\hline \multirow[t]{2}{*}{ PDLG } & 0.9 & 0.05 & -0.04 & -0.06 \\
\hline & & 0.01 & -0.05 & -0.07 \\
\hline
\end{tabular}

a $\Delta \mathrm{p} K_{\mathrm{a}}=\left(\mathrm{p} K_{\mathrm{a}}\right)_{\mathrm{ex}}-\left(\mathrm{p} K_{\mathrm{a}}\right)_{\mathrm{th}}$.

rations and obtained a value of $3.0 \mathrm{kcal} \mathrm{mol}^{-1}$ as a non-electrostatic factor in $\Delta G$ at $\alpha=1.0$. They attributed this to differential hydration between the polymer and the monomer counterpart. The $\Delta \mathrm{p} K_{\mathrm{a}}$ values for PAA at $\alpha=0.6$ are larger than those for PDLG at $\alpha=0.9$ which has the same axial charge density as the former. This may be because the higher the surface charge density, the closer the condensed counterions are to the polymer charges, resulting in the higher degree of dehydration. The $\Delta \mathrm{p} K_{\mathrm{a}}$ values in Table III are independent of $C_{\mathrm{s}}$ in the region studied here. This is understood by the fact that the first hydration layer around small ions is hardly affected by the ionic strength in such a low $C_{\mathrm{s}}$ region. ${ }^{20}$

\section{CONCLUDING REMARKS}

So far, many potentiometric titration curves of linear polyions have been discussed in terms of models ${ }^{12,21-23}$ other than condensation theories; one of the models ${ }^{21}$ is a rod or a cylinder model with smeared charge and utilizes the Poisson-Boltzmann equation. Another ${ }^{12,22,23}$ is a discrete site model which considers neighbor interactions like Ising model. Such models can fit experimental data on $\mathrm{p} K_{\mathrm{a}}$, though they need adjustable parameters such as cylinder radius, closest ap- 
proach distance and interaction parameters among neighboring functional groups. With the latter model, in particular, it is possible to include the contribution from non-electrostatic interactions such as hydration change into the interaction parameters.

In the present study, we applied IMM, a modified condensation theory free from adjustable parameters, to the potentiometric titration of linear polyacids. We obtained a smooth sigmoidal titration curve by IMM. The underestimation of $\mathrm{p} K_{\mathrm{a}}$ at the lower charge density suggests a limitation of the linear charge array model adopted in the condensation theories. We interpreted $\Delta \mathrm{p} K_{\mathrm{a}}$ for PAA at the higher charge density as being caused by factors other than the longrange electrostatic interaction. This interpretation was rationalized, though indirectly, by facts that IMM can predict qualitatively the dependence of $\mathrm{p} K_{\mathrm{a}}$ of PAA on $C_{\mathrm{p}}{ }^{1 / 2}$, and that the deviation of the theoretical $\mathrm{p} K_{\mathrm{a}}$ from the experimental data at lower $\alpha$ region can be corrected via the estimation of the effective charge densities which uses experimental and the theoretical $\gamma_{c}{ }^{\prime}$ values.

CPM proved to fail in predicting the increasing trend of $\mathrm{p} K_{\mathrm{a}}$ with $\alpha$. This was ascribed to the neglect of the mixing entropy of the condensed counterions. Thus we conclude that the combination of the site binding-like binding mode and the averaged reduction of effective polymer charge due to the counterion condensation is necessary for the analysis of the properties of polyelectrolytes in solutions based upon the condensation concept. In our forthcoming paper, ${ }^{24}$ we will extend IMM to systems containing counterions with different valences.

\section{REFERENCES}

1. M. Satoh, T. Kawashima, J. Komiyama, and T. Iijima, Polym. J., 19, 1191 (1987).

2. G. S. Manning, J. Chem. Phys., 51, 924 (1969).

3. K. Iwasa and J. C. T. Kwak, J. Phys. Chem., 81, 408 (1977).

4. G. S. Manning, J. Phys. Chem., 85, 870 (1981).

5. D. S. Olander and A. Holtzer, J. Am. Chem. Soc., 90, 4549 (1968).

6. M. Nagasawa, T. Murase, and K. Kondo, J. Phys. Chem., 69, 4005 (1965).

7. J. C. Fenyo, F. Delben, S. Paoletti, and V. Crescenzi, J. Phys. Chem., 81, 1900 (1977).

8. M. Nagasawa and A. Holtzer, J. Am. Chem. Soc., 86, 538 (1964).

9. N. Murai and S. Sugai, Biopolymers, 13, 857 (1974).

10. M. Kowblansky and P. Zema, Macromolecules, 14, 166 (1981).

11. Y. M. Joshi and J. C. T. Kwak, J. Phys. Chem., 83, 1978 (1979).

12. A. Minakata, K. Matsumura, S. Sasaki, and H. Ohnuma, Macromolecules, 13, 1549 (1980).

13. E. A. Lewis, T. J. Barkley, R. R. Reams, and L. D. Hansen, Macromolecules, 17, 2874 (1984).

14. A. Ikegami, J. Polym. Sci., A., 2, 907 (1964).

15. M. Satoh, M. Hayashi, J. Komiyama, and T. Iijima, to be submitted.

16. M. Arshadi, R. Yamdagni, and P. Kebarle, J. Phys. Chem., 74, 1475 (1970).

17. H. G. Hertz and M. D. Zeidler, Ber. Bunsenges. Phys. Chem., 68, 821 (1964).

18. H. G. Hertz, Angew. Chem. Int. Edit. English, 9, 124 (1970).

19. Y. Marcus, "Introduction to Liquid State Chemistry," Wiley, New York, 1977.

20. J. O. M. Bockris and P. P. S. Saluja, J. Phys. Chem., 76, 2140 (1972).

21. L. Kotin and M. Nagasawa, J. Chem. Phys., 36, 873 (1962).

22. S. Sasaki and A. Minakata, Biophys. Chem., 11, 199 (1980).

23. R. L. Cleland, Macromolecules, 17, 634 (1984).

24. M. Satoh, T. Kawashima, and J. Komiyama, to be submitted. 